\title{
Efficient Bound for Conditional Min-Entropy of Physical Unclonable Functions Beyond IID
}

\author{
Florian Wilde and Christoph Frisch and Michael Pehl \\ Technical University of Munich \\ Munich, Germany \\ \{florian.wilde, chris.frisch, m.pehl\}@tum.de
}

\begin{abstract}
The remaining min-entropy of a secret generated by fuzzy extraction from a Physical Unclonable Function is typically estimated under the assumption of independent and identically distributed PUF responses, but this assumption does not hold in practice. This work analyzes the more realistic case that the responses are independent but not necessarily identically distributed. For this case, we extend the (n-k) bound and a tighter bound by Delvaux et al. In particular, we suggest a grouping bound which provides a trade off for accuracy vs computational effort. Comparison to previous bounds shows the accuracy and efficiency of our bound. We also adapt the key rank (a tool from side-channel analysis) to cross-validate the state-of-the-art and our proposed min-entropy bounds based on publicly available PUF data from real hardware.
\end{abstract}

\section{INTRODUCTION}

With the demand for security in low-cost devices, Physical Unclonable Functions (PUFs) raised attention as a cheap and still secure alternative for key storage compared to secured non-volatile memory (NVM). PUFs utilize variations of the manufacturing process. These variations cause unpredictable and uncontrollable differences in the behavior of identical circuits on different positions on a certain chip and on different chips. The PUF response, a chip-unique secret which is not permanently stored but derived by the PUF on demand, is obtained from such differences. This work focuses on PUFs comprised of multiple PUF cells, each contributing one bit to the PUF response. The expected value of a chip's PUF response is fixed during the manufacturing process. Its value, however, may vary between measurements due to noise, environmental effects, and aging. Hence the PUF response cannot be used directly as a key. The typical approach to obtain a key from a PUF is to (i) measure the noisy PUF response, (ii) map it to a noisy codeword of an error correcting code (ECC) by means of helper data and a helper data algorithm (HDA), and (iii) decode via ECC to derive a stable key.

The helper data may be stored unprotected and are generated during a roll-out phase, usually in a code-offset fuzzy extractor [1] or fuzzy commitment [2] scheme. This schemes generate helper data $\vec{y}=\vec{x} \oplus \vec{w}$ by encoding a random $k$ bit vector $\vec{r}$ to an $n$ bit codeword $\vec{w}$ and XORing an $n$ bit PUF response $\vec{x}$ to $\vec{w}$. Depending on the scheme, the secret $\vec{s}$, which is hashed to the actual key, is either $\vec{x}$ or $\vec{r}$. For performance reasons

WIFS'2019, December, 9-12, 2019, Delft, Netherlands. 978-1-7281-3217-4/19/\$31.00 (C)2019 IEEE.

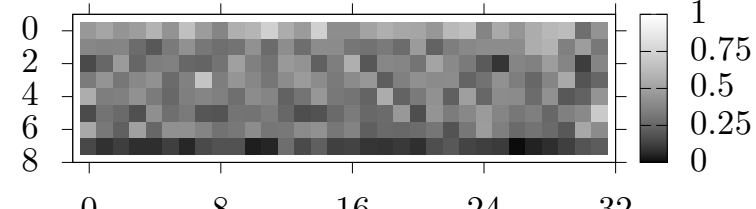

$\begin{array}{lllll}0 & 8 & 16 & 24 & 32\end{array}$

Figure 1. Relative frequency of 1 (Bit-Alias) for the PUF bits derived from the dataset [4]. Squares reflect the positions of PUF cells (RO pairs) on a device.

the key is usually split into multiple chunks, so an ECC with smaller message length can be used.

For the schemes and under the assumption of bias-free and independent and identically distributed (IID) PUF responses, the entropy of the key, when neglecting the hash, is limited by $k$ [3]. Most real world PUFs do not produce bias-free responses, though; even worse: they do not even fit to an IID assumption. Despite great efforts to design PUF cells for unbiased output, incautious layout, unexpected influence of adjacent logic, or small deviations in mask production can already lead to biased responses. Because these effects differ for each position on the die, the resulting bias is not identical. An example is shown in Fig. 1 The heat map shows for each cell of an RO PUF the relative frequency $p$ for the corresponding response bit to turn out as a 1 among all devices in the dataset (i.e. the Bit-Alias [5]). The underlying RO frequencies are taken from [4]; a response bit is derived by mutually exclusive pairwise comparions between two adjacent ROs in a row. Previous work shows that this approach produces responses almost entirely free of spatial correlation [6], but cannot remove the observable bias, which can be explained by placement and routing [7]. Similar effects with varying impact are frequently observable in PUF implementations.

An attacker who tries to reveal the key stored by a PUF knows the helper data and the type of ECC. Thus, leveraging the Bit-Aliases gives the attacker a significant advantage when guessing the key. The effort for guessing the key in such a setting might be approximated by guesswork and its relatives [8]-[10]. But this is not feasible for the PUF setting due to its high computational complexity. Another tool to bound the quality of a key is entropy. Due to the cryptographic relevance, only min-entropy is considered throughout this work.

Contribution: Estimation of the key entropy given known helper data was previously studied in [1], [11]. However, these works only cover the case of biased IID and unbiased 
correlated PUF responses. This work first summarizes the main achievements in the state of the art and demonstrates the problems, which are caused when (i) not considering the blockwise processing during post-processing, or (ii) assuming IID instead of independent but not necessarily identically distributed (IND) PUF responses. Several improvements upon previous approaches are presented. In particular, we introduce the grouping bound, which provides a practically feasible bound of the conditional min-entropy of IND PUF responses. All improvements are demonstrated using real world PUF data. Additionally, the entropy estimates are compared to the key rank, i.e. the actual effort for guessing a key.

Structure: After discussing the state of the art of entropy estimation for PUFs in Sec. II we introduce our improvements in Sec. III Results are provided and analyzed in the context of key rank in Sec. IV. Sec. V] concludes our work.

\section{StATE-OF-ThE-ART ENTROPy ESTIMATION FOR PUFs}

To evaluate the security of a PUF based key storage, the conditional entropy of the PUF response $\vec{x}$ given the helper data $\vec{y}$ is the important figure of merit. This section presents state-of-the-art methods to calculate or bound the conditional entropy, but first discusses entropy estimation of the PUF alone as a necessary prerequisite.

\section{A. PUF without Helper Data}

Typically, response bits of PUFs are considered realizations of IID Bernoulli random variables. I.e. each bit takes the value 1 with probability $p$ independent of all other bits. Under this assumption, and given a sufficiently large number of observed bits, if necessary from multiple devices, $p$ is well approximated by the relative frequency of a 1 in the data set. Then the min-entropy $m$ of a device's $n$ bit PUF response $\vec{x}$ is

$$
m=H_{\infty}(X)=-n \log _{2}(\max (p, 1-p)) .
$$

However, as already pointed out in Sec. I for many PUF implementations the probability for a 1 differs significantly between the positions on the die as shown in Fig. 1. This contradicts the IID assumption so that (1) becomes imprecise.

Consequently, Wilde et al. [12] assumed the bits of the PUF responses to be IND. In this case, the probabilities for a 1 form a vector $\vec{p}=\left(\begin{array}{lll}p_{1} & \cdots & p_{n}\end{array}\right)$, where $p_{i}$ is approximated by the relative frequency of a 1 among the bits originating from position $i$ on the die. The min-entropy under the IND assumption is

$$
\tilde{m}=\tilde{H}_{\infty}(X)=-\sum_{i=1}^{n} \log _{2}\left(\max \left(p_{i}, 1-p_{i}\right)\right) .
$$

The extent of the variation in entropy per bit, together with the approximated entropy per bit under the IID assumption, is depicted in Fig. 2. For most positions, the entropy under IID assumption is severely overestimated compared to the IND case, which sums up to 240 bit instead of 188 bit over all positions, cf. Tbl. I] The root cause for the difference in

\footnotetext{
${ }^{1}$ All vectors in this work are row vectors unless explicitly stated otherwise.
}

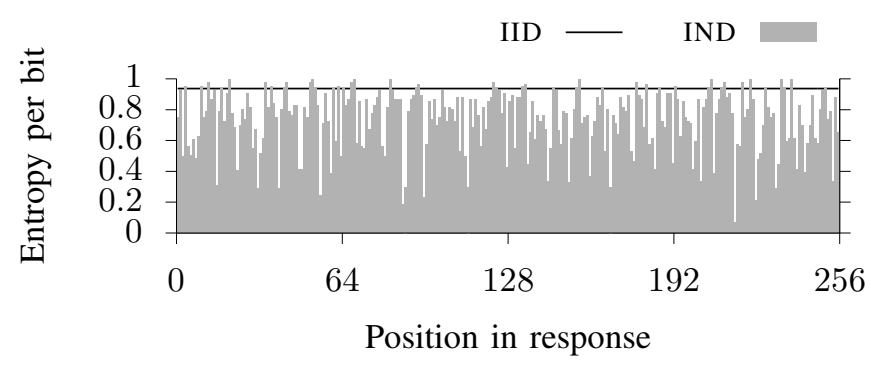

Figure 2. Min-entropy per bit for PUF response $\vec{x}$ under IID assumption and for IND case. IID uses 11 without multiplication by $n$ and with $p=\sum p_{i} / n$. IND uses 2] without summation.

entropy estimates is visible in Fig. 1. Only few positions have $p_{i} \approx 0.5$, while most positions suffer from either $p_{i}>0.5$ or $p_{i}<0.5$, i.e. a bias towards 1 or towards 0 . Both cases cause a reduction in entropy $\tilde{m}$ according to (2). In the IID case, however, $p$ equals the average over all $p_{i}$, which causes those $p_{i}>0.5$ to partially cancel out with those $p_{i}<0.5$, resulting in $p$ being incorrectly close to 0.5 and overestimating entropy.

\section{B. (n-k) Bound}

Given the entropy of the PUF alone, an easy-to-compute and frequently used, yet pessimistic, lower bound for the conditional entropy of the PUF response given the helper data is the (n-k) bound [1]. It applies only to the IID case, where it calculates the entropy estimate $l$ of an $o$ bit key $\vec{s}$ by a simple balance sheet: Ingoing to the fuzzy extractor are $m$ bit of entropy from the PUF response $\vec{x}$ and $k$ bit of entropy from a random number $\vec{r}$ that has full entropy. Outgoing are $n$ bit of entropy for the helper data $\vec{y}$. An additional loss of $L$ bit occurs in the hash function that compresses the fuzzy extractor output into a fixed length key.

$$
l=m+k-n-L
$$

Neglecting the hash function, the entropy loss between $\vec{x}$ and $\vec{s}$ is $n-k$, which gave the bound its name.

This approach can result in negative values for $l$, cf. Tbl. I because $n-k$ is only an upper bound for the entropy loss that holds with equality only if $m=n$ [11].

\section{Average Conditional Min-Entropy}

Instead of the pessimistic (n-k) bound, Delvaux et al. [11] provide a direct mathematical expression for the conditional entropy based on the code-offset fuzzy extractor described by Dodis et al. [13]. Evaluation is only feasible for small codes [11], but it provides a baseline to compare the bounds against. By application of Bayes' rule to the average conditional minentropy definition in [13] they yield

$$
H_{\infty}(X \mid Y)=-\log _{2}\left(\frac{1}{|\mathcal{R}|} \sum_{\vec{y} \in \mathcal{Y}} \max _{\vec{w} \in \mathcal{W}} \mathrm{P}(X=\vec{y} \oplus \vec{w})\right)
$$

for an ECC in general and respectively the more efficient

$$
H_{\infty}(X \mid Y)=-\log _{2}\left(\sum_{\vec{\epsilon} \in \mathcal{E}} \max _{\vec{w} \in \mathcal{W}} \mathrm{P}(X=\vec{\epsilon} \oplus \vec{w})\right)
$$


for linear ECCs. Here $\mathcal{R}=\{0,1\}^{1 \times k_{b}}$ is the set of possible messages of the ECC and $\mathcal{Y}=\{0,1\}^{1 \times n_{b}}$ the set of possible helper data generated for one ECC block. $\vec{w}$ is a codeword from the codeword space $\mathcal{W}$ and $\vec{\epsilon}$ an element of the minimum Hamming-weight error vector space $\mathcal{E}$ such that $\{\vec{w} \oplus \vec{\epsilon} \mid \vec{w} \in \mathcal{W}, \vec{\epsilon} \in \mathcal{E}\}=\mathcal{Y}$, i.e. $\vec{\epsilon}$ is a coset leader. Using a 3 -repetition code as example, $\mathcal{W}=\{000,111\}$ and $\mathcal{E}=\{000,001,010,100\}$.

Equations (4) and (5) are not limited to block codes [11], but in such a case, they are applied on a single ECC block only. For the IID case considered in [11], it is sufficient to multiply the result with the number of ECC blocks required to produce the entire secret $\vec{s}$ to get an overall result.

\section{Efficient Lower Bound for Average Conditional Min- Entropy for Large $n_{b}$}

Because (4), (5) require up to $2^{n_{b}}|\mathcal{R}|$ or respectively $2^{n_{b}}$ operations, direct evaluation even for moderately sized codes such as a $(63,7,15) \mathrm{BCH}$-code is hardly feasible. Therefore, [11] proposed an algorithm that reduces computational effort via groups of PUF responses with the same probability, sorted in decreasing order of probability, to avoid computations that will later be discarded by the max operator in (4), (5).

Under the IID assumption, all $2^{n_{b}}$ responses can be grouped into $J=n_{b}+1$ groups $\varphi_{j}$ with probability

$$
q_{j}=q^{j}(1-q)^{n_{b}-j}
$$

where $j \in\left[0, n_{b}\right]$ and $q=\min (p, 1-p)$. Each $\varphi_{j}$ then contains

$$
\left|\varphi_{j}\right|=\left(\begin{array}{c}
n_{b} \\
j
\end{array}\right)
$$

PUF responses $\vec{x}$ with probability $q_{j}$ and

$$
q_{j}>q_{j+1}
$$

This is useful because, for a given $\vec{y}$, the max operator in (4), (5) selects the most likely $\vec{x}$ that is reachable by addition of a $\vec{w}$. Therefore, and because $\vec{r}$ is chosen uniformly, $|\mathcal{R}|=|\mathcal{W}|$ elements of $\mathcal{Y}$ map to the same $\vec{x}$ as best guess, cf. Fig. 3 . E.g. for $\vec{y}=100$ and $\vec{y}=011, \vec{x}=100$ is the most likely $\vec{x}$ within reach via $\vec{w}=000, \vec{w}=111$. It is thus sufficient to consider $2^{n_{b}} /|\mathcal{R}|$ (or $2^{n_{b}-k_{b}}$ for block codes) elements of $\mathcal{X}$ to accumulate the best guesses for all $\vec{y} \in \mathcal{Y}$.

Which $2^{n_{b}} /|\mathcal{R}|$ elements of $\mathcal{X}$ to consider depends on the specific ECC. For repetition codes with odd $n_{b}, \bigcup_{j=0}^{t} \varphi_{j}$, i.e. the $2^{n_{b}} /|\mathcal{R}|$ most likely $\vec{x}$, are the correct choice, because due to the IID assumption they either equal $\mathcal{E}$ for $p<0.5$, cf. Fig. 3, or, if $p>0.5$, can be mapped to $\mathcal{E}$ by addition of a fixed $\vec{w}$. However, for a $(15,5,3) \mathrm{BCH}$-code, in addtion to $\bigcup_{j=0}^{3} \varphi_{j}, 420 \vec{x}$ from $\varphi_{4}$ and 28 from $\varphi_{5}$ are required. By instead choosing $448 \vec{x}$ from $\varphi_{4}$, thus again the $2^{n_{b}} /|\mathcal{R}|$ most likely $\vec{x}$, we overestimate the probability for 28 out of $1024 \vec{x}$. Always choosing the $2^{n_{b}} /|\mathcal{R}|$ most likely $\vec{x}$ thus provides an upper bound for the sum in (4), (5), which leads to a lower bound for $H_{\infty}(X \mid Y)$ that holds with equality for maximum distance separable (MDS) ECCs.

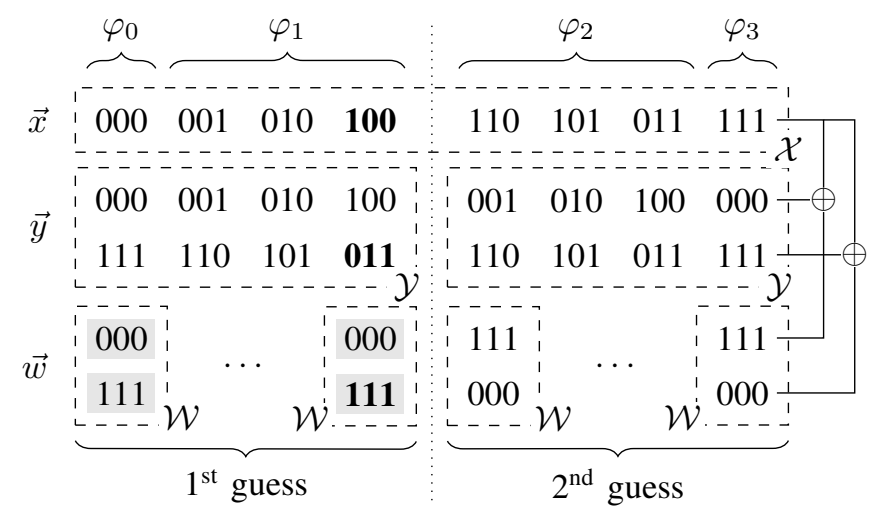

Figure 3. Visualization of the algorithm from [11] using a 3-repetition code and $p<0.5$. The best guess - which is the one selected by the max operator in (4), 5 - for every possible $\vec{y}$ can be found in the left half of the figure. E.g. for $\vec{y}=011$, the best guess is to assume $\vec{x}=100$ thus $\vec{w}=111, \vec{r}=1$.

As $q_{j}$ is equal for all $\vec{x}$ in a $\varphi_{j},\left|\varphi_{j}\right|$ responses $\vec{x}$ can be processed at once. Because $\left|\varphi_{j}\right|$ often exceeds $|\mathcal{R}|$ already for small $j$, this provides the most speed up, so that for a linear $\left(n_{b}, k_{b}, t\right)$ block code, $t$ to $t+1$ computations suffice.

To summarize, this approach results in a much tighter lower bound for the remaining min-entropy than the (n-k) bound and is feasible in practice if the IID assumption holds.

\section{IMPROVED BLOCKWISE CONDITIONAL ENTROPY ESTIMATIONS FOR THE IND CASE}

In this section, we present improved entropy bounds for keys derived from PUFs. They are developed under the constraint of practical applicability and consider that PUF response bits are (i) IND rather than IID and (ii) usually processed blockwise.

\section{A. (n-k) Bound}

To bring the (n-k) bound to the IND case, $m$ is to be replaced by $\tilde{m}$ from (2). However, this only uses a better estimation for the ingoing entropy from the PUF, while the entropy loss is still at its worst-case. Hence the exact entropy according to (4) is significantly underestimated, cf. Tbl. I] which might cause overdesign and, therefore, high costs.

A tighter, yet very fast to calculate bound is found when considering blockwise processing of PUF bits. The blocks are independent of each other even in the IND case. Therefore, one block cannot leak information about another and entropy can be estimated per block. Fig. 4 shows the estimated entropy per bit given $p_{i}$ values according to Fig. 1, bounds are calculated for the complete PUF response under the IID and the IND assumption as well as blockwise assuming IND and a 5repetition code. The actual entropy per block is obviously bound from below by zero. Thus, the blockwise estimate of the overall entropy is

$$
\tilde{l}=\sum_{i=1}^{N_{b}} \max \left(\tilde{m}_{i}+k_{b}-n_{b}, 0\right)
$$

where $\tilde{m}_{i}$ is the entropy in the PUF bits processed in block $i$; the number of blocks for encoding a complete key 
Table I

ESTIMATIONS AND BOUNDS FOR MIN-ENTROPY OF $\vec{x}$ AND FOR CONDITIONAL MIN-ENTROPY OF $\vec{s}$ FOR (N)-REP AND (N,K,T)-BCH CODES

\begin{tabular}{|c|c|c|c|c|c|c|c|c|c|c|c|}
\hline & \multirow[b]{2}{*}{$n$} & \multirow[b]{2}{*}{$m$} & \multirow[b]{2}{*}{$\tilde{m}$} & \multirow[b]{2}{*}{$k$} & & \multicolumn{2}{|c|}{$n-k)$ bound } & \multicolumn{2}{|c|}{ avg. cond. min-entropy } & \multicolumn{2}{|c|}{ Grouping, $\theta_{\Delta}=$} \\
\hline & & & & & $l$ & $l(\tilde{m})$ & $\tilde{l}$ & IID & IND & 0.05 & 0.10 \\
\hline & 256 & 240 & 188 & & & & & & & & \\
\hline (3) & 255 & 239 & 187 & 85 & 69.0 & 17.3 & 22.4 & 77.1 & 47.2 & 45.9 & 43.9 \\
\hline (5) & 255 & 239 & 187 & 51 & 35.0 & -16.7 & 3.30 & 45.1 & 22.5 & 21.7 & 19.6 \\
\hline (7) & 252 & 236 & 185 & 36 & 20.2 & -30.7 & 0.01 & 31.2 & 12.5 & 11.9 & 10.6 \\
\hline (21) & 252 & 236 & 185 & 12 & -3.78 & -54.7 & 0.00 & 9.38 & 1.56 & 1.25 & 0.86 \\
\hline$(7,4,1$ & 252 & 236 & 185 & 144 & 128 & 77.3 & 77.3 & 132 & 88.4 & 85.1 & 79.5 \\
\hline$(15,5,3)$ & 255 & 239 & 187 & 85 & 69.0 & 17.3 & 17.3 & 76.2 & 40.6 & 37.3 & 33.0 \\
\hline$(31,6,7)$ & 248 & 232 & 182 & 48 & 32.5 & -18.1 & 0.00 & $N a N$ & $N a N$ & 13.3 & 10.7 \\
\hline$(63,7,15)$ & 252 & 236 & 185 & 28 & 12.2 & -38.7 & 0.00 & $N a N$ & $N a N$ & 3.82 & 2.36 \\
\hline$(127,8,31)$ & 254 & 238 & 186 & 16 & 0.09 & -51.6 & 0.00 & $N a N$ & $N a N$ & 0.57 & 0.19 \\
\hline
\end{tabular}

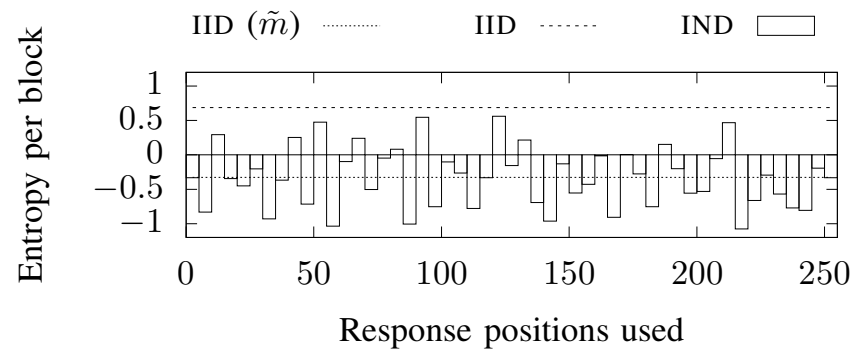

Figure 4. Min-entropy per block for $\vec{s}$ for a 5-repetition code using original (n-k) bound and improved version 9. For IID, i.e. using $m$ in (3), $l / N_{b}$ is overestimated; $l(\tilde{m}) / N_{b}$ is a too pessimistic bound. IND shows the entropy estimate per block $\tilde{m}_{i}+k_{b}-n_{b}$.

is $N_{b}=\left\lfloor\frac{n}{n_{b}}\right\rfloor$. Each block encodes $k_{b}$ disjoint bits from the random number $\vec{r}$, which is assumed to have $k=k_{b} \cdot N_{b}$ bits of entropy, and contributes $n_{b}$ bits of helper data to $\vec{y}$. Tbl. II shows the improvement through the tightened bound for real world data [4] and different codes.

\section{B. Average Conditional Min-Entropy}

Under the IID assumption, it is sufficient to apply (4) or (5) on one block. The exact entropy in the key is then derived by multiplying the result with the number of required codewords $N_{b}$. To extend this exact approach to the IND case, we compute the entropy individually for all $N_{b}$ blocks, using the accurately estimated probabilities in $\vec{p}$ to get $\mathrm{P}(X=\vec{\epsilon} \oplus \vec{w})$. The entropies per block derived with (5) under the IID and IND assumption are depicted in Fig. 5 and listed in Tbl. [ Both show that falsely making an IID assumption severely overestimates the remaining entropy, which puts the security of the overall system at risk.

\section{The Grouping Bound: An Efficient Lower Bound for Aver- age Conditional Min-Entropy for Large $n_{b}$}

For the IND case, which is not considered in [11], no two responses might have equal probability. If so, grouping according to [11] ends up in $2^{n_{b}}$ groups $\varphi_{j}$ with $\left|\varphi_{j}\right|=1$. Consequently, the final reduction from $2^{n_{b}} /|\mathcal{R}|$ to $t$ or $t+1$ computations is no longer applicable, which makes computation infeasible for large codes. In the following, we propose a new algorithm to calculate a strict lower bound (or optionally

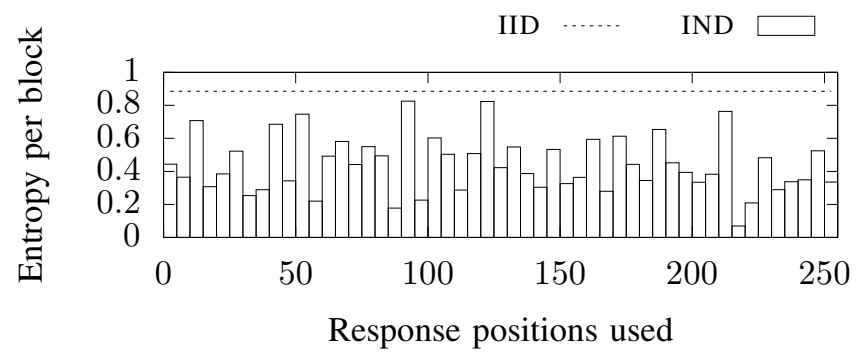

Figure 5. Min-entropy per block for $\vec{s}$ for a 5-repetition code using original and blockwise average conditional min-entropy. As with the (n-k) bound, the IID assumption severely overestimates entropy compared to the IND case.

an approximation) for the average conditional min-entropy for the IND case in feasible time.

First, assume that for all $p_{i}$ in $\vec{p}$

$$
\forall i: p_{i} \geq 0.5 \text {. }
$$

Practically, this is achieved by setting each $p_{i}$ that violates the assumption to $1-p_{i}$ and inverting the corresponding helper data bit. Because $H_{\infty}(p)=H_{\infty}(1-p)$, entropy is unaffected and the entropy estimation obtained from the preprocessed data holds for the original data. For the special case where, after the transform, $\exists 0 \leq p_{a} \leq 1:\left(\forall i:\left(p_{i} \approx p_{a}\right)\right)$, which fits for some SRAM PUFS [14], sufficiently accurate results may be obtained under the IID assumption with $p$ set to $p_{a}$.

For the general IND case, though, a method is needed to identify and calculate the probability of the $2^{n_{b}} /|\mathcal{R}|$ most likely PUF responses $\vec{x}$ efficiently. The key to this are large response groups $\varphi_{j}$, because all $\vec{x}$ in a group $\varphi_{j}$ can be covered by a single computation. $\left|\varphi_{j}\right|$ is strongly affected by the number of unique values in $\vec{p}$, because if some $p_{i}$ are equal, the probability of an $\vec{x}$ only depends on how many, but not which, of the corresponding response bits are flipped in $\vec{x}$, creating a large number of $\vec{x}$ to be put into the same $\varphi_{j}$. Due to the fast increase of the binomial coefficient, this gain is already large for a small number of equal $p_{i}$.

We therefore suggest to trade off accuracy against computational effort: The $p_{i}$ are collected in $T$ bias groups $\rho_{\tau}$ such that the difference between any two $p_{i}$ within any $\rho_{\tau}$ is at most $\theta_{\Delta}$. All $\eta_{\tau} p_{i}$ within a group $\rho_{\tau}$ are assigned a representative probability $\theta_{\tau}$ and $\vec{\theta}=\left(\begin{array}{lll}\theta_{1} & \cdots & \theta_{T}\end{array}\right)$. The value of $\theta_{\tau}$ may 
be the maximum of all $p_{i}$ in the group to obtain a strict lower bound for the remaining entropy, or some kind of average, e.g. mean or median, to obtain an approximation of the remaining entropy. Changing $\theta_{\Delta}$ trades off tightness of the bound against computational cost, because it equals the maximum error made by approximation of some $p_{i}$ and influences $\left|\varphi_{j}\right|$ via $\eta_{\tau}$. Independent of the chosen $\theta_{\Delta}$, all response bits have to belong to exactly one bias group $\rho_{\tau}$, thus

$$
n_{b}=\sum_{\tau=1}^{T} \eta_{\tau} .
$$

After the transition from $\vec{p}$ to $\vec{\theta}$, the probability of an $\vec{x}$ only depends on the number of flipped bits from each bias group $\rho_{\tau}$. Hence, a response group $\varphi_{j}$, which contains all $\vec{x}$ with same probability, is uniquely described by the number $\zeta_{j, \tau}$ of bits flipped from each group $\rho_{\tau}$ or by a flip vector $\overrightarrow{\zeta_{j}}=\left(\begin{array}{lll}\zeta_{j, 1} & \cdots & \zeta_{j, T}\end{array}\right)$. Assume this vectors to be stored in a $J \times T$ matrix

$$
\check{Z}=\left(\begin{array}{lll}
\vec{\zeta}_{0}^{\prime} & \cdots & \vec{\zeta}_{J}^{\prime}
\end{array}\right)^{\prime},
$$

which is sorted according to (8). Transposition of a vector is indicated in this work by a'. The number of rows in $\check{Z}$ then equals the number of response groups $\varphi_{j}$,

$$
J=\prod_{\tau=1}^{T}\left(\eta_{\tau}+1\right),
$$

and each group $\varphi_{j}$ contains

$$
\left|\varphi_{j}\right|=\prod_{\tau=1}^{T}\left(\begin{array}{c}
\eta_{\tau} \\
\zeta_{j, \tau}
\end{array}\right)
$$

PUF responses $\vec{x}$ with same probability $q_{j}$. We summarize this group sizes as $\vec{\psi}=\left(\left|\varphi_{0}\right| \cdots\left|\varphi_{J}\right|\right)$. Because of (10), the best guess is $\vec{x}=\overrightarrow{1}=\left(\begin{array}{lll}1 & \cdots & 1\end{array}\right)$, which corresponds to $\overrightarrow{\zeta_{0}}=\overrightarrow{0}$, and

$$
q_{0}=\prod_{\tau=1}^{T} \theta_{\tau}^{\eta_{\tau}},
$$

which can be written in the log-domain as

$$
\varsigma_{0}=\log _{2}\left(q_{0}\right)=\vec{\eta} \log _{2}(\vec{\theta})^{\prime}
$$

using a scalar product of two vectors. Based on $\varsigma_{0}$, the logprobabilities $\varsigma_{j}$ of all response groups $\varphi_{j}$ follow from

$$
\vec{\varsigma}^{\prime}=\overrightarrow{1}^{\prime} \varsigma_{0}+\check{Z}\left(\log _{2}(\overrightarrow{1}-\vec{\theta})-\log _{2}(\vec{\theta})\right)^{\prime} .
$$

To eventually bound the min-entropy, only the first $\Omega$ elements from $\vec{\psi}$ and $\vec{\varsigma}$ are necessary. $\Omega$ is the smallest $j$ such that $\sum_{i=0}^{j}\left|\varphi_{i}\right| \geq 2^{n_{b}} /|\mathcal{R}|$. Due to (14), $\Omega<J \ll 2^{n_{b}} /|\mathcal{R}|$. Then the grouping bound is

$$
H_{\infty}(X \mid Y) \leq-\log _{2}\left(\vec{\psi} \exp _{2}(\vec{\varsigma})^{\prime}\right) .
$$

The resulting bounds for several $\theta_{\Delta}$ and codes, even for larger codes such as $(127,8,31)-\mathrm{BCH}$, are listed in Tbl. [1 Note that when setting $\theta_{\tau}$ to the lowest $p_{i}$ in a bias group $\rho_{\tau}$, the resulting estimate $H_{\infty}^{L}(X \mid Y)$ is no bound for entropy because the algorithm still uses the $2^{n_{b}} /|\mathcal{R}|$ most likely PUF responses. However, for $H_{\infty}^{H}(X \mid Y)$ with $\theta_{\tau}$ set to the highest $p_{i}$ in a bias group, $H_{\infty}^{L}(X \mid Y)-H_{\infty}^{H}(X \mid Y)$ is an upper bound for the error made through the quantization of probabilities.

Remark: Until now we neglected to describe how to derive the sorted matrix $\check{Z}$, a task which is equivalent to computing how many bits have to be flipped from the bias groups $\rho_{\tau}$ for each of the $2^{n_{b}} /|\mathcal{R}|$ most likely PUF responses. This is a non-trivial task and falls into the category of integer linear programming, which is NP-hard and would require to iterate over $J$ groups $\varphi_{j}$ in the worst case.

However, a function can be written that, given a target range of probability, returns the flip vectors $\vec{\zeta}_{j}$ that result in such a probability by recursion within the $\rho_{\tau}$. Once sorted, the returned vectors constitute a continuous part of $\check{Z}$. The first $\Omega$ rows of $\check{Z}$ can thus be assembled from repeated calls to the function, starting with a target range from the probability of the best guess downwards, adding the results for consecutive target ranges until a sufficient part of $\check{Z}$ is obtained.

\section{VAlidating Entropy Bounds With Key Rank}

The previous sections analyzed state-of-the-art methods and explained our extentions for the IND case to estimate or lower bound the conditional min-entropy of a PUF response given the helper data. We claimed that because the state-of-the-art tools only apply to the IID case, users are forced to take this assumption, even if not justified, thereby jeopardizing the security of the system due to a severe overestimation of minentropy. The corresponding results are summarized in Tbl. []

In this section, we aim to validate these results by simulating actual attacks on a code-offset construction using the 192 devices that constitute the dataset from [4], which has been used throughout this work. We randomly chose $10^{4} 144$ bit keys (the maximum storable key length with the considered codes on our device) and implemented them on each device, using all codes mentioned in Tbl. I, to obtain corresponding helper data. Because a device provides 256 response bits, only $k=k_{b}\left\lfloor\frac{256}{n_{b}}\right\rfloor$ bits of the key can be stored for an $\left(n_{b}, k_{b}, t\right)$ ECC. Where $k<144$, we used the first $k$ bits of the key and the first $n=n_{b} \frac{k}{k_{b}}$ bits of the PUF response. The entropy contained in these first $n$ response bits (not given any helper data) under the IID and IND assumption is reported in Tbl. I in columns $m, \tilde{m}$. Given the helper data and the Bit-Alias $\vec{p}$, we determined the key rank for each key, device, and ECC.

The metric key rank originates from the field of sidechannel analysis. It represents the number of unsuccessful guesses an attacker would make until the correct key is found while following an optimal guessing strategy. To find the optimal guessing strategy, one needs information on which key hypotheses are more likely. In side-channel analysis, such information results from e.g. measuring the electro-magnetic emanations of a device while it performs a cryptographic operation. For PUFs, the Bit-Alias provides such information.

\footnotetext{
${ }^{2}$ MATLAB code available at https://gitlab.lrz.de/tueisec/ind_puf_entropy
} 
An attacker may obtain a sufficiently accurate estimation of it by purchasing (as legitimate customer) a sufficient number of devices from the same type and analyzing them in detail. To calculate the key rank even for ECCs where $2^{k}$ is infeasible to enumerate, we use the method by Glowacz et al. [15].

Fig. 6reports the logarithmic key rank in bit for each device as a histogram for each ECC. The key rank depends on the device, but not the key, because the guessing strategy aims at the PUF response and once it is correctly guessed, the helper data provide a direct mapping to the key. Vertical bars with a cross indicate $k$, as $2^{k}$ is the number of all possible guesses for a $k$ bit key. Vertical bars with a circle indicate the result of the grouping bound for $\theta_{\Delta}=0.05$, i.e. a lower bound for $H_{\infty}(X \mid Y)$. According to NIST, $H_{\infty}-1$ bit provides a lower bound for average key rank if at most $k^{*}<k$ guesses are made per device [10]. Hence an attacker must expect to require at least $2^{H_{\infty}-1}$ guesses on average to find the correct key.

The validity of the bounds under IID assumption is already falsified in Tbl. \ by comparison to the exact average conditional min-entropy with our extension for IND. Comparison with the key rank shows that the (n-k) bound under IND assumption is unpractically conservative. The results for our grouping bound, however, are all safely below the average key rank without being too conservative. This confirms that our work provides a valid bound to assess the security of a PUF based key storage using the common code-offset construction.

For the grouping bound itself, a trade off between accuracy and runtime complexity is observable: Compared to the actual average conditional min-entropy, where it is feasible to compute, $\theta_{\Delta}=0.05$ provides the tighter bound. However, it also requires a longer runtime due to the determination of the most likely flip vectors $\vec{\zeta}_{j}$ : For the computationally most expensive case of a $(127,8,31)$ BCH-code, the runtime on a commodity computer is $\approx 20 \mathrm{~min}$ for $\theta_{\Delta}=0.05$, compared to $\approx 10 \mathrm{~s}$ for $\theta_{\Delta}=0.1$. In either case, our grouping bound is the tightest bound under IND assumption that is feasible for large codes.

\section{CONCLUSION}

To verify the security of a PUF based key storage, the remaining conditional entropy of the key is crucial. However, current methods are either inaccurate or infeasible without an IID assumption for the PUF response. Because the IID assumption is not justified for many types of PUF, this work proposes an accurate and feasible method to lower bound the remaining entropy under less stringent IND assumption. Results of applying the bound to different codes and for real world data show the quality of the bound. The results also demonstrate the relation between the bound and the average key rank, i.e. the effort an attacker must expect for guessing the key under an optimal guessing strategy.

\section{ACKNOWLEDGMENT}

This work was partly funded by the German Federal Ministry of Education and Research in the project HQS through grant number 16KIS0616. Permanent ID and revision date of this document: 3b0bccc028d2b79fcd67c668263243ce13b6112d 2019-10-02

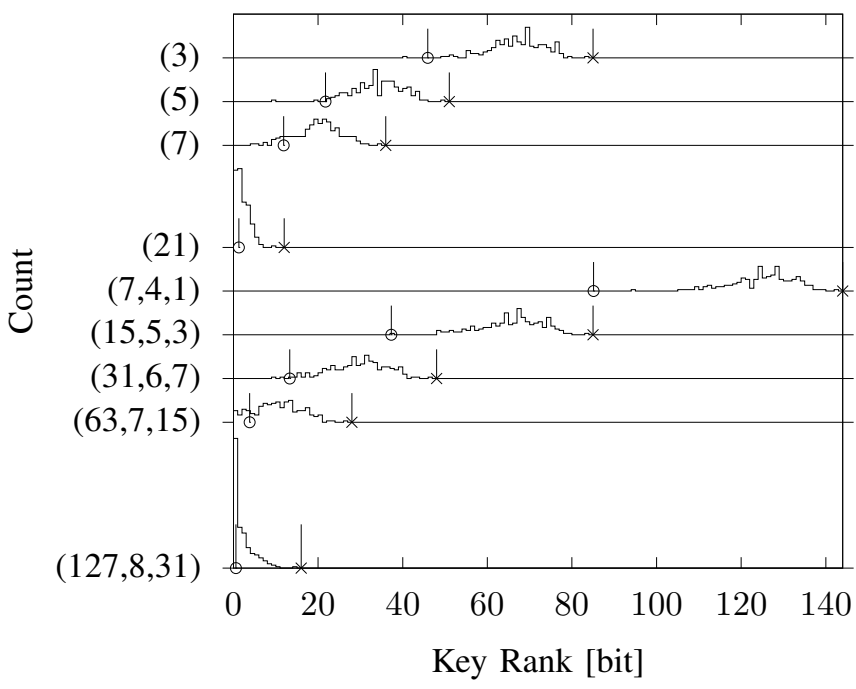

Figure 6. Histogram of key rank in bit for all devices in the Maiti et al. dataset. Vertical lines indicate the grouping bound for $\theta_{\Delta}=0.05$ and the theoretical maximum $k$, e.g. $2 \cdot 8$ bit $=16$ bit for the $(127,8,31) \mathrm{BCH}$-code.

\section{REFERENCES}

[1] Y. Dodis, L. Reyzin, and A. Smith, "Fuzzy extractors: How to generate strong keys from biometrics and other noisy data," in Advances in Cryptology - EUROCRYPT 2004, C. Cachin and J. L. Camenisch, Eds. Springer Berlin Heidelberg, 2004, pp. 523-540.

[2] A. Juels and M. Wattenberg, "A fuzzy commitment scheme," in ACM Conference on Computer and Communications Security (CCS). ACM, 1999, pp. 28-36.

[3] M. Pehl, M. Hiller, and G. Sigl, Information Theoretic Security and Privacy of Information Systems. Cambridge University Press, 2017, ch. Secret Key Generation for Physical Unclonable Functions, pp. 362389.

[4] A. Maiti. (2011) PUF download data. Virginia Tech. Accessed 201709-27. [Online]. Available: http://rijndael.ece.vt.edu/puf/download.html

[5] A. Maiti et al., "A large scale characterization of RO-PUF," in Hardware-Oriented Security and Trust (HOST), 2010 IEEE International Symposium on. IEEE, 2010, pp. 94-99.

[6] F. Wilde, B. M. Gammel, and M. Pehl, "Spatial correlation analysis on physical unclonable functions," IEEE Transactions on Information Forensics and Security, vol. 13, no. 6, pp. 1468-1480, June 2018.

[7] M. Pehl, M. Hiller, and H. Graeb, "Efficient evaluation of physical unclonable functions using entropy measures," Journal of Circuits, Systems and Computers, vol. 25, no. 01, p. 1640001, 2016.

[8] J. L. Massey, "Guessing and entropy," in Information Theory, Proceedings., 1994 IEEE International Symposium on. IEEE, 1994, p. 204.

[9] J. O. Pliam, "On the incomparability of entropy and marginal guesswork in brute-force attacks," in Progress in Cryptology INDOCRYPT 2000 B. Roy and E. Okamoto, Eds. Springer, 2000, pp. 67-79.

[10] M. S. Turan et al., Recommendation for the Entropy Sources Used for Random Bit Generation. NIST, 2018.

[11] J. Delvaux et al., "Efficient fuzzy extraction of PUF-induced secrets: Theory and applications," IACR Cryptology ePrint Archive, vol. 2015, no. 854,2015 .

[12] F. Wilde, M. Hiller, and M. Pehl, "Statistic-based security analysis of ring oscillator PUFs," in Integrated Circuits (ISIC), 2014 14th International Symposium on. IEEE, 2014, pp. 148-151.

[13] Y. Dodis et al., "Fuzzy extractors: How to generate strong keys from biometrics and other noisy data,' SIAM Journal on Computing, vol. 38 , no. 1, pp. 97-139, 2008.

[14] F. Wilde, "Large scale characterization of SRAM on Infineon XMC microcontrollers as PUF," in 4th Workshop on Cryptography and Security in Computing Systems (CS2 2017) HIPEAC17, Stockholm, 12017.

[15] C. Glowacz et al., "Simpler and more efficient rank estimation for sidechannel security assessment," in Fast Software Encryption, G. Leander, Ed. Springer Berlin Heidelberg, 2015, pp. 117-129. 